\title{
Non-Invasive Characterization of Cardiac Activation Patterns: Contributions of Body Surface Potential Mapping in Healthy Volunteers
}

\author{
João Salinet ${ }^{1}$, Jimena G S Paredes ${ }^{1}$, Marcelo Mazzetto ${ }^{2}$, Victor G Marques ${ }^{1}$, Nelson Samesima ${ }^{3}$, \\ Carlos A Pastore ${ }^{3}$, Idágene A Cestari ${ }^{2}$ \\ ${ }^{1}$ Biomedical Engineering, Engineering, Modelling and Applied Social Sciences Centre, Federal \\ University of ABC, Brazil \\ ${ }^{2}$ Bioengineering Division, Heart Institute (InCor), Hospital das Clinicas HCFMUSP, Faculdade de \\ Medicina, Universidade de Sao Paulo, Sao Paulo, Brazil \\ ${ }^{3}$ Clinical Unit of Electrocardiography, Heart Institute (InCor), Hospital das Clinicas HCFMUSP, \\ Faculdade de Medicina, Universidade de Sao Paulo, Sao Paulo, Brazil
}

\begin{abstract}
Body surface potential mapping (BSPM) systems allow non-invasive investigation of the spatial-temporal behaviour of cardiac electrical activity. This study aims to present the validation (application) of 62-channel BSPM equipment. 12-lead ECG plus two leads on the back were recorded (21 healthy volunteers) and further segmented for 4 consecutive beats allowing to obtain $P$, $Q R S$ and $T$ peaks and heart activity $R-R, P R, Q R S, S T$ and $Q T$ segments. The vectorcardiograms (VCG) are extracted from the electrodes placed on the torso (direct measurement - DM) or indirectly by the Inverse Dower, Uijen and Willems methods. 17 instants of time during one heart beat (P-QRS-T) (Figure 2) are used to generate sequential isopotential maps for each healthy volunteer to investigate propagation of highest and lowest potentials presented on each map. The results obtained in healthy volunteers are comparable with results in the literature, suggesting the system can help identifying heart rhythm disorders, in patients.
\end{abstract}

\section{Introduction}

Invasive and non-invasive commercial systems have been used in clinical practice to help diagnose and treat heart arrhythmia patients [1]. Recently, body surface potential mapping (BSPM) systems have been used with great expectation to estimate epicardium electrical activity, increasing the success rate of treatment of patients with complex heart disorders [2]. BSPM systems allow real time acquisition of up to 300 electrodes with the body surface potentials (BSPs) projected with a colour-coded basis on a 3D torso shell, allowing non-invasive investigation of the spatial-temporal behaviour of the cardiac electrical activity. Recently, we described improvements on a pseudo real time BSPM system with 62-channel for non-invasive investigation of the heart's electrical activity $[3,4]$. In this work we present the application of the method developed in humans. In this study, we report results in. healthy volunteers

\section{Methods}

\subsection{Electrocardiographic study}

Sixty-two body surface electrodes (35 on the chest, 8 on the sides of the torso, and 19 on the back) (Figure 1) are used to record 2 minutes of non-invasive electrical heart activity from 21 volunteers with no previous history of cardiovascular diseases. Data were collected after a 5-min rest in the sitting position (ethics committee research protocol $\mathrm{n}^{\circ}$ CAAE:-00591712.0.1001.0068).

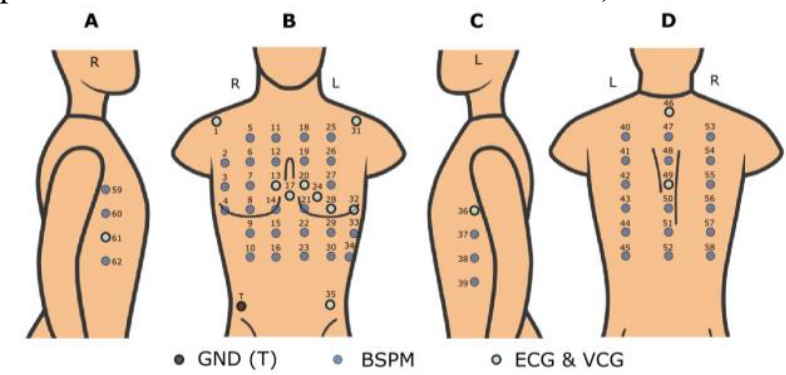

Figure 1: Spatial distributions of the body surface electrodes; (A) Right-side view; (B) Anterior-posterior view; (C) Left-side view; (D) Posterior-anterior view.

\subsection{Electrocardiographic analyses}

Body surface signals (BSPs) are sampled at $1 \mathrm{kHz}$ and pre-processed with a $2^{\text {nd }}$ order Savitzky-Golay filter with cut-off frequency at $150 \mathrm{~Hz}$ [7], notch filter at $60 \mathrm{~Hz}$ [8]. Baseline wandering is removed by subtracting the output 
of a second order Lynn's lowpass filter from the delayed output [5], eliminating frequencies below $0.26 \mathrm{~Hz}$.

\section{2-Lead ECG segmentation}

The 12-lead ECG was obtained from the BSPs. Segmentation consists of selecting 4 consecutive beats and then identifying the peaks and onset-offset from the $\mathrm{P}, \mathrm{QRS}$ and $\mathrm{T}$ waves throughout recently validated methods $[6,7,8]$. Leads 48 and 49 are also considered to show correlation with left atrium activity [9].

\section{Vectorcardiogram}

The vectorcardiograms (VCG) are extracted from the electrodes placed on the torso (direct measurement - DM) with their respective Frontal, Horizontal and Sagittal planes generated, based on Frank's lead system [10], where three orthogonal leads, corresponding to the axes of the body ( $\mathrm{Vx}, \mathrm{Vy}$ and $\mathrm{Vz})$, are calculated based on potential values in predetermined torso's leads position [1]. The orthogonal leads are also obtained indirectly through a weighted average on selected leads of the 12lead ECG. We implemented the weighting transfer coefficients applied to preselected leads from three distinct methods, Inverse Dower (ID), Uijen (UI) and Willems (WI) [1]. The size and direction of vector's propagation from the loops related to the $\mathrm{P}, \mathrm{QRS}$ and $\mathrm{T}$ waves are investigated. The frontal, horizontal and sagittal planes obtained from DM are compared with ID, UI and WI indirect methods. Comparison between methods takes into account loop morphology, quadrant location and orientation.

\section{Body surface isopotential maps}

17 instants of time during one heartbeat (P-QRS-T) (Figure 2) are used to generate sequential isopotential maps for each healthy volunteer to investigate the propagation of highest and lowest potentials presented on each map. During the atrial activity, 4 different instants of time are represented by the dashed vertical lines between a and d, comprising the onset of atrial excitation and its recovery within a $100 \mathrm{~ms}$ window. Ventricle excitation is evaluated in 8 different time instants also within $100 \mathrm{~ms}$ (e to l), and ventricles repolarization (segment ST and T wave) by 5 different moments ( $m$ to $q)$.

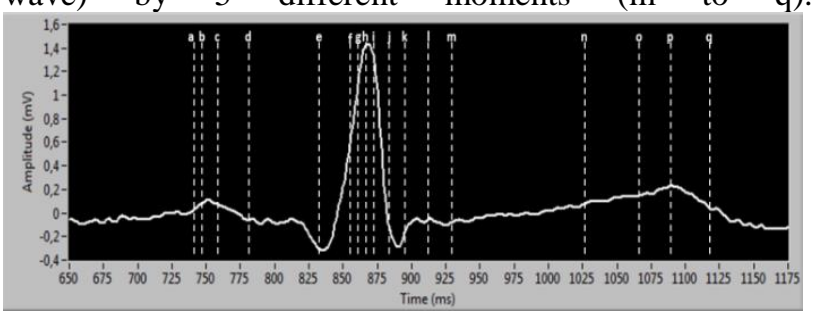

Figure 2: Different time instants chosen during a heartbeat to observe the propagation of potentials through the generation of isopotential maps at those moments.

\section{Results and Discussions}

Volunteers were all male, with a mean age of $27 \pm 6$ years, IMC of $23.96 \pm 3.06$, being most on regular exercise.

\section{ECG segmentation}

Figure 3 highlights one example of the ECG segmentation (lead II). The analysis was expanded for all volunteers and is summarized in Table 1.

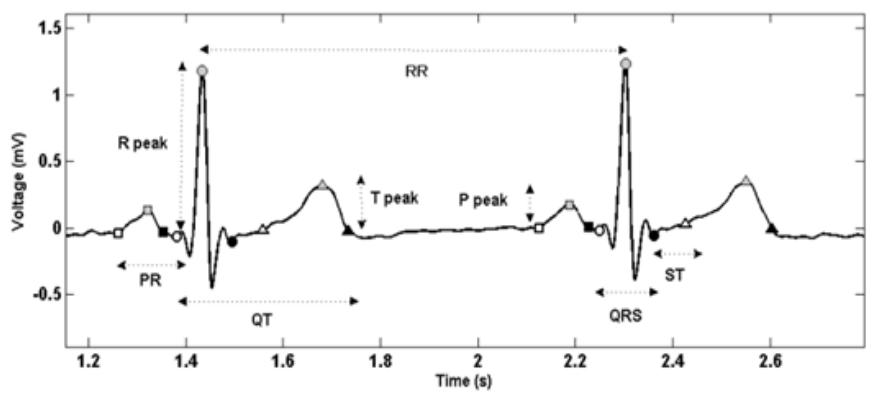

Figure 3: Lead II segmentation of a volunteer. Square, circle and triangle markers refer to $\mathrm{P}, \mathrm{QRS}$ and $\mathrm{T}$ wave respectively. Markers in white, grey and black means the onset, peak and off-set respectively for each heart beat wave.

\section{VCG characterization}

The $\mathrm{P}$ loop presented anti-clockwise rotation in $67 \%$ and $57 \%$ of the cases in the frontal and horizontal planes respectively. For the sagittal plane the majority had clockwise direction (67\%). For the QRS loop, 80.95\% of the volunteers had a clockwise rotation in the frontal plane, $100 \%$ and $95 \%$ anti-clockwise rotation in the horizontal and sagittal planes respectively. For the $\mathrm{T}$ wave loop, 90.47\% had clockwise rotation in the frontal plane, 95\% and $85.71 \%$ anti-clockwise rotation in the horizontal and sagittal planes. QRS loop was found in $95 \%$ of cases in the 4th quadrant in the frontal plane, $95 \%$ in the 1st quadrant in the horizontal plane, and $80.95 \%$ in the 4th quadrant in the sagittal plane.

For the P loop, in $38 \%$ of the cases, it was not possible to identify quadrant location due to noise influence on low signals amplitude. Of the remainder, $52 \%$ and $76.19 \%$ were located in the 4th quadrant in the frontal and sagittal planes respectively, and $61.90 \%$ in the 1 st quadrant in the horizontal plane. With respect to the $\mathrm{T}$ curve, it is observed that $95 \%$ of the cases were presented in the 4th quadrant both in the frontal and horizontal planes, and finally in $90 \%$ of the volunteers, the curve was presented in the third quadrant in the sagittal plane. 
Table 1: Parameters obtained from a 12-Lead ECG recording (Leads 48 and 49)

\begin{tabular}{|c|ccc|ccccc|}
\cline { 2 - 8 } \multicolumn{1}{c|}{} & \multicolumn{3}{c|}{ Peak amplitude (mV) } & \multicolumn{4}{c|}{ Interval (ms) } \\
\cline { 2 - 9 } \multicolumn{1}{c|}{} & $\mathbf{P}$ & $\mathbf{R}$ & $\mathbf{T}$ & $\mathbf{R}-\mathbf{R}$ & $\mathbf{P R}$ & $\mathbf{Q R S}$ & $\mathbf{S T}$ & QT \\
\hline I & $0.01 \pm 0.02$ & $-0.01 \pm 0.03$ & $0.12 \pm 0.02$ & $780.34 \pm 26.85$ & $138.19 \pm 20.41$ & $89.38 \pm 15.06$ & $69.85 \pm 20.53$ & $334.01 \pm 13.04$ \\
II & $0.07 \pm 0.02$ & $1.25 \pm 0.05$ & $0.23 \pm 0.02$ & $776.52 \pm 24.33$ & $154.71 \pm 17.17$ & $85.33 \pm 13.23$ & $83.46 \pm 28.39$ & $345.18 \pm 14.88$ \\
II & $0.07 \pm 0.02$ & $1.08 \pm 0.06$ & $0.10 \pm 0.03$ & $765.60 \pm 50.07$ & $150.77 \pm 16.16$ & $87.54 \pm 14.29$ & $89.70 \pm 28.74$ & $348.55 \pm 18.10$ \\
\hline $\mathbf{A} \mathbf{V}_{\mathbf{R}}$ & $-0.03 \pm 0.02$ & $-0.62 \pm 0.03$ & $-0.17 \pm 0.01$ & $772.09 \pm 44.53$ & $152.32 \pm 22.59$ & $86.28 \pm 14.06$ & $70.65 \pm 18.86$ & $340.99 \pm 15.49$ \\
$\mathbf{A V}_{\mathbf{L}}$ & $-0.04 \pm 0.02$ & $-0.53 \pm 0.04$ & $-0.01 \pm 0.02$ & $759.29 \pm 50.30$ & $146.22 \pm 26.08$ & $90.82 \pm 15.63$ & $114.54 \pm 27.68$ & $353.08 \pm 19.68$ \\
$\mathbf{A V}_{\mathbf{F}}$ & $0.07 \pm 0.02$ & $1.10 \pm 0.07$ & $0.16 \pm 0.02$ & $769.53 \pm 72.86$ & $140.39 \pm 30.68$ & $96.81 \pm 27.50$ & $76.18 \pm 20.90$ & $351.24 \pm 27.72$ \\
\hline $\mathbf{V}_{\mathbf{1}}$ & $0.00 \pm 0.01$ & $-0.74 \pm 0.07$ & $0.04 \pm 0.02$ & $781.36 \pm 53.75$ & $133.42 \pm 13.73$ & $157.90 \pm 67.29$ & $40.51 \pm 94.52$ & $347.46 \pm 33.51$ \\
$\mathbf{V}_{\mathbf{2}}$ & $0.01 \pm 0.02$ & $-1.36 \pm 0.10$ & $0.28 \pm 0.03$ & $781.98 \pm 21.73$ & $154.33 \pm 19.14$ & $90.15 \pm 7.24$ & $61.82 \pm 18.93$ & $375.06 \pm 49.73$ \\
$\mathbf{V}_{\mathbf{3}}$ & $-0.02 \pm 0.02$ & $-1.39 \pm 0.11$ & $0.37 \pm 0.02$ & $782.63 \pm 18.45$ & $137.30 \pm 13.47$ & $95.06 \pm 9.55$ & $55.96 \pm 16.49$ & $330.02 \pm 7.32$ \\
$\mathbf{V}_{\mathbf{4}}$ & $-0.02 \pm 0.02$ & $-1.21 \pm 0.08$ & $0.45 \pm 0.03$ & $783.08 \pm 21.73$ & $154.17 \pm 16.40$ & $90.51 \pm 7.61$ & $60.58 \pm 12.51$ & $330.48 \pm 9.89$ \\
$\mathbf{V}_{\mathbf{5}}$ & $-0.02 \pm 0.02$ & $-0.56 \pm 0.05$ & $0.45 \pm 0.03$ & $781.33 \pm 25.02$ & $157.24 \pm 13.03$ & $74.50 \pm 31.07$ & $73.47 \pm 36.16$ & $330.47 \pm 7.03$ \\
$\mathbf{V}_{\mathbf{6}}$ & $-0.01 \pm 0.02$ & $0.16 \pm 0.04$ & $0.31 \pm 0.02$ & $764.68 \pm 49.46$ & $152.37 \pm 17.64$ & $84.09 \pm 7.81$ & $72.92 \pm 16.67$ & $334.71 \pm 7.59$ \\
\hline $\mathbf{V}_{\mathbf{4 8}}$ & $0.00 \pm 0.02$ & $0.07 \pm 0.05$ & $-0.06 \pm 0.02$ & $779.00 \pm 24.47$ & $181.56 \pm 38.19$ & $93.37 \pm 11.42$ & $73.97 \pm 19.85$ & $344.74 \pm 10.71$ \\
$\mathbf{V}_{\mathbf{4 9}}$ & $0.01 \pm 0.02$ & $0.01 \pm 0.03$ & $-0.09 \pm 0.02$ & $773.88 \pm 74.97$ & $157.95 \pm 59.83$ & $98.01 \pm 12.75$ & $68.58 \pm 26.00$ & $341.94 \pm 21.44$ \\
\hline
\end{tabular}

A typical example of a comparison between VCG methods is highlighted in Figure 4. All methods are in the same quadrant across the planes, and with the same direction of rotation. In terms of the QRS loop morphology, UI showed greater similarity with DM. This analysis was expanded to the whole population and the following was observed: in the frontal plane, the highest visual similarity was obtained between DM and ID methods (57\% of the cases), while in the horizontal and sagittal planes in $85.71 \%$ and $71.42 \%$ there was higher similarity between DM and EU methods. In $>80 \%$ of cases, the corresponding QRS loop is in the same quadrant as the DM method. Through visual inspection, it was observed that the frontal plane showed the lowest similarity values, with $66 \%$ in the case of UI and WI when compared with DM. A higher similarity (> 80\%) was observed between ID and EU methods. There was no significant variation in terms of quadrant loop location across the methods, and direction disagrees in $20 \%$ of the cases (especially in the frontal and sagittal planes). Finally, UI and WI were similar in around $61 \%$ in the frontal plane. Both the location of the curve and its direction showed no major changes, except for the sagittal plane that shows a $71 \%$ similarity.

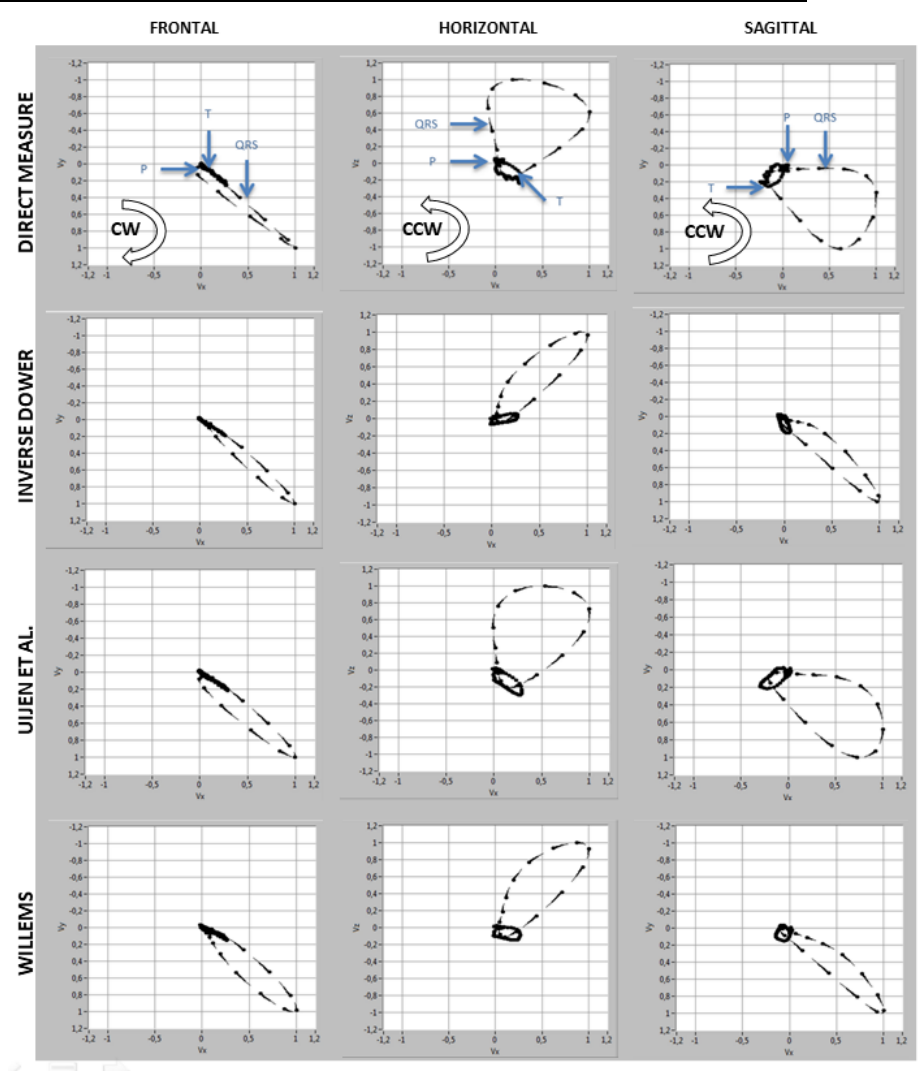

Figure 4: Typical example of the VCG methods from of a volunteer.

\section{Isopotential Maps}

The most common pattern seen in the study population regarding the propagation of the maxima and minima of the P, QRS and T wave isopotential maps is 
highlighted in Figure 5. During the P and QRS, regions that are negative reflect the perspective of receding depolarization surfaces, whereas positive approaching depolarization surfaces. For $\mathrm{T}$ wave, both polarity and interpretation are reversed.

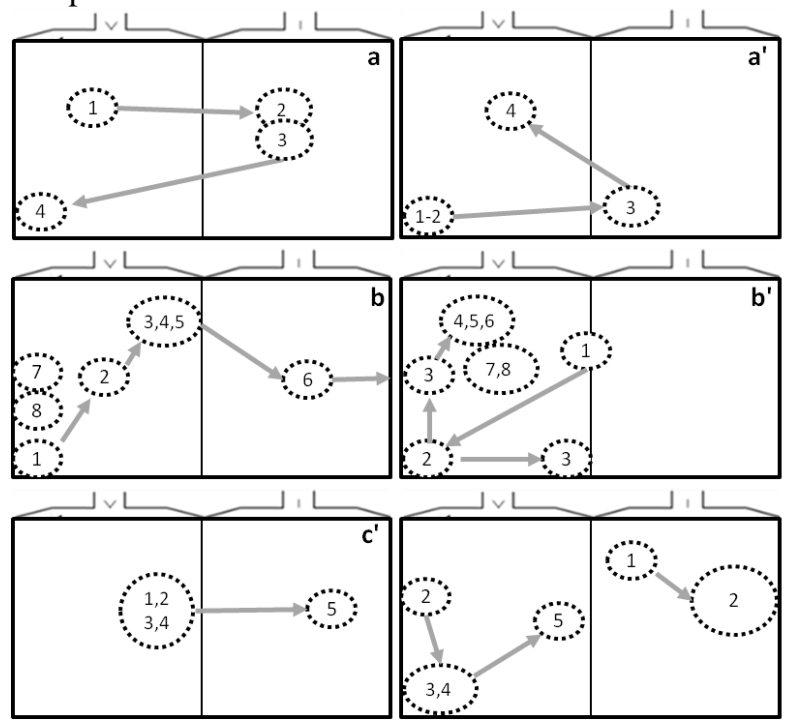

Figure 5: Propagation of the highest and lowest potentials during the atrial and ventricular activity and repolarization.

\section{Conclusions}

A non-invasive investigation tool using high density electrodes was validated in healthy male volunteers. Results demonstrate the applicability of the method to investigate the behaviour from the heart of patients suffering from heart rhythm disorders.

\section{Acknowledgements}

Financial support: São Paulo State Research Foundation (FAPESP grant 2012/50283-6 and scholarship 2016/26240-6 to VGM). JGSP is funded by the Program of Alliances for Education and Training (Scholarship Brazil - PAEC OEA-GCUB-2017).

\section{References}

[1] Macfarlane PW, Van Oosterom A, Pahlm O, Kligfield P, Janse M, Camm J. Comprehensive Electrocardiology. Springer Science \& Business Media, 2010.

[2] Cluitmans MJM, Bonizzi P, Karel JMH, et al. In Vivo Validation of Electrocardiographic Imaging. JACC: Clinical Electrophysiology

[3] Salinet JL, Camargo EDLB, Meneghing CEB,
Cestari IA . Plataforma para Mapeamento de Biopotenciais do Torso em Alta Densidade. Anais do XXV Congresso Brasileiro de Engenharia Biomédica (CBEB 2016), 2016. p. 1678-1682.

[4] Salinet JL, Marques, VG, Mazzetto M, Camargo EDLB, Pastore CA, Cestari IA. A 64-lead Body Surface Potential Mapping System. In: 44th annual scientific meeting of Computing in Cardiology (CinC), 2017, Rennes. Computing in Cardiology 2017; Vol 44:1:4. ISSN: 2325-887X DOI:10.22489/CinC.2017.053-337.

[5] Kozumplík J, Provazník I. Fast Time-Varying Linear Filters for Suppression of Baseline Drift in Electrocardiographic Signals. Biomedical Engineering Online 2017;16(1):24.

[6] Madeiro JP, Cortez P, Marques JA, VázquezSeisdedos CR, Sobrinho CRMR. An Innovative Approach of QRS Segmentation Based on FirstDerivative, Hilbert and Wavelet Transforms. Medical Engineering \& Physics 2012; 34(9): 1236-1246.

[7] Madeiro JP, Nicolson WB, Cortez PC, Marques JA, Vázquez-Seisdedos $\mathrm{CR}$, Elangovan $\mathrm{N}, \mathrm{Ng}$ GA, Schlindwein FS. New Approach for TWave Peak Detection and T-Wave End Location in 12-lead Paced ECG Signals Based on a Mathematical Model. Med Eng Phys. 2013;35(8):1105-15.

[8] Madeiro JP, Santos EMBE, Cortez PC, Felix JHDS, Schlindwein FS. Evaluating Gaussian and Rayleigh-Based Mathematical Models for $\mathrm{T}$ and P-waves in ECG. IEEE Latin America Transactions 2017; 15(5): 843-853.

[9] Bojarnejad M, Blake JR, Bourke J, Shepherd E, Murray A, Langley P. Non-Invasive Estimation of Left Atrial Dominant Frequency In Atrial Fibrillation From Different Electrode Sites: Insight from Body Surface Potential Mapping. J Atr Fibrillation. 2014 Oct-Nov.

[10] Frank E. An Accurate, Clinically Practical System for Spatial Vectorcardiography. Circulation 1956;13(5):737-749.

[11] Pastore CA, Samesima N, Tobias N, Filho HG. Eletrocardiografia Atual - Curso do Serviço de Eletrocardiogradia do InCOr; 2016. 3a. ed., Atheneu.

Address for correspondence

Dr João Salinet

Biomedical Engineering - CECS- UFABC

Email: joao.salinet@ufabc.edu.br 